\title{
Alternativas en gestión local del riesgo en cantones sin plan regulador: caso de Alajuelita.
}

\author{
Alternatives in local risk management in cantons without \\ regulatory plan: case of Alajuelita
}

\author{
Luis Nelson Arroyo-González ${ }^{1}$ \\ Universidad Nacional, Costa Rica
}

\begin{abstract}
Resumen
La situación de deterioro ambiental y la ausencia de un plan regulador ha motivado el establecer alternativas de gestión del riesgos a pobladores de algunos vecindarios mediante charlas, capacitaciones, entrega de material informativo y prácticas de campo. Ello ha llevado a la formación de comités de emergencias vecinales, en donde la incorporación de pobladores ha establecido una diferencia sustancial en la identificación, vigilancia y atención de sitios de riesgo, con mejoras evidentes en la organización comunal y el autocuidado.
\end{abstract}

Palabras clave: Gestión del riesgo, amenazas naturales, organización comunal.

\begin{abstract}
The situation of environmental deterioration and the absence of a Regulatory Plan, has motivated the establishment of Risk Management alternatives to residents of some neighborhoods through talks, trainings, delivery of informative material and field practices. This has led to the formation of Neighborhood Emergency Committees, where the incorporation of residents has established a substantial difference in the identification, surveillance and attention of risky sites, with evident improvements in the community organization and self-care.
\end{abstract}

Keywords: Risk management, natural threats, community organization

1 Académico e investigador, Escuela de Ciencias Geográficas, Universidad Nacional, Costa Rica. Correo electrónico: luis.arroyo.gonzalez@una.cr

Este artículo corresponde a la ponencia presentada en el I Congreso Centroamericano de Ciencias de la Tierra y el Mar, realizado en San José, Costa Rica, del 13 al 16 de noviembre de 2017. 


\section{Introducción}

En el año 2016 dio inicio el proyecto denominado "Capacidades municipales y comunitarias en gestión del riesgo", mediante la Agencia de Cooperación Internacional del Japón (JICA) en conjunto con el Centro de Prevención de Desastres Naturales de América Central (CEPREDENAC) y la Comisión de Emergencias de Costa Rica (CNE). Este acuerdo de cooperación es un componente del Programa BOSAI Fase II de JICA, el cual forma parte del Proyecto para el Desarrollo de Capacidades para la Gestión de Riesgos a Desastres en América Central, y que se desarrolla en Costa Rica con el propósito de fortalecer las capacidades municipales y comunitarias para mejorar la información sobre amenazas y desarrollar capacidades para la reducción de los riesgos ante desastres.

Se ha denominado a este proyecto como BOSAI-II, por cuanto el BOSAI-I se desarrolló en Costa Rica durante los años 2007-2012. En este periodo las actividades que efectuó tuvieron como escenario la provincia de Guanacaste, específicamente, en el cantón de Cañas, en donde se llevaron a cabo tareas significativas en la preparación para desastres en la comunidad de Hotel, así como en otros vecindarios ubicados en la cuenca baja del río Cañas.

En estas localidades se establecieron procedimientos concretos basados en actividades orientadas a promover la prevención y preparación para desastres, a través de una serie de tareas pendientes en donde, además de la formación de líderes locales, se promovió el enfoque solidario, basado en la identificación de ciudadanos con "deseos de reducir el riesgo a desastres, a través de la adopción de experiencias, lecciones aprendidas y aprendizaje, que evidenció en buenas prácticas de reducción comunitaria de los riesgos". (JICA, CEPREDENAC, CNE, 2007-2012)

\section{Área de estudio y aspectos demográficos generales}

El cantón de Alajuelita es una unidad administrativa con una extensión de $21,2 \mathrm{Km}^{2}$ que se localiza inmediatamente al sur de la ciudad de San José, ocupando una serie de formaciones geológicas de topografía montañosa. (Mapa 1) A finales del siglo XIX esta y otras localidades conformaban hábitats dispersos, que se integraban en esencia, con base al cultivo del café. Ello varió a partir de la concentración de la actividad económica y poblacional que convierte la Región Central en el núcleo de 
mayor presencia poblacional y de servicios, ya que la Gran Área Metropolitana cubre el 3,8\% del territorio y alberga cerca del $60 \%$ de la población del país. Como parte de esta tendencia, la densidad de habitantes por kilómetro cuadrado en este cantón se multiplicó por siete en relación con el 2011 y con respecto a 1963. Durante este lapso de tiempo se mantiene este crecimiento en forma homogénea en términos de no aumentos sensibles, situación que se modifica a partir de 1984, cuando en el censo de población de 2000 la población experimentó un crecimiento de cerca de 100000 habitantes. Para el año 2015 se contabilizaban ya 87734 habitantes. (Véase gráfico No.1). (INEC, 2011).

Mapa 1. Ubicación área de estudio, cantón de Alajuelita, San José, Costa Rica

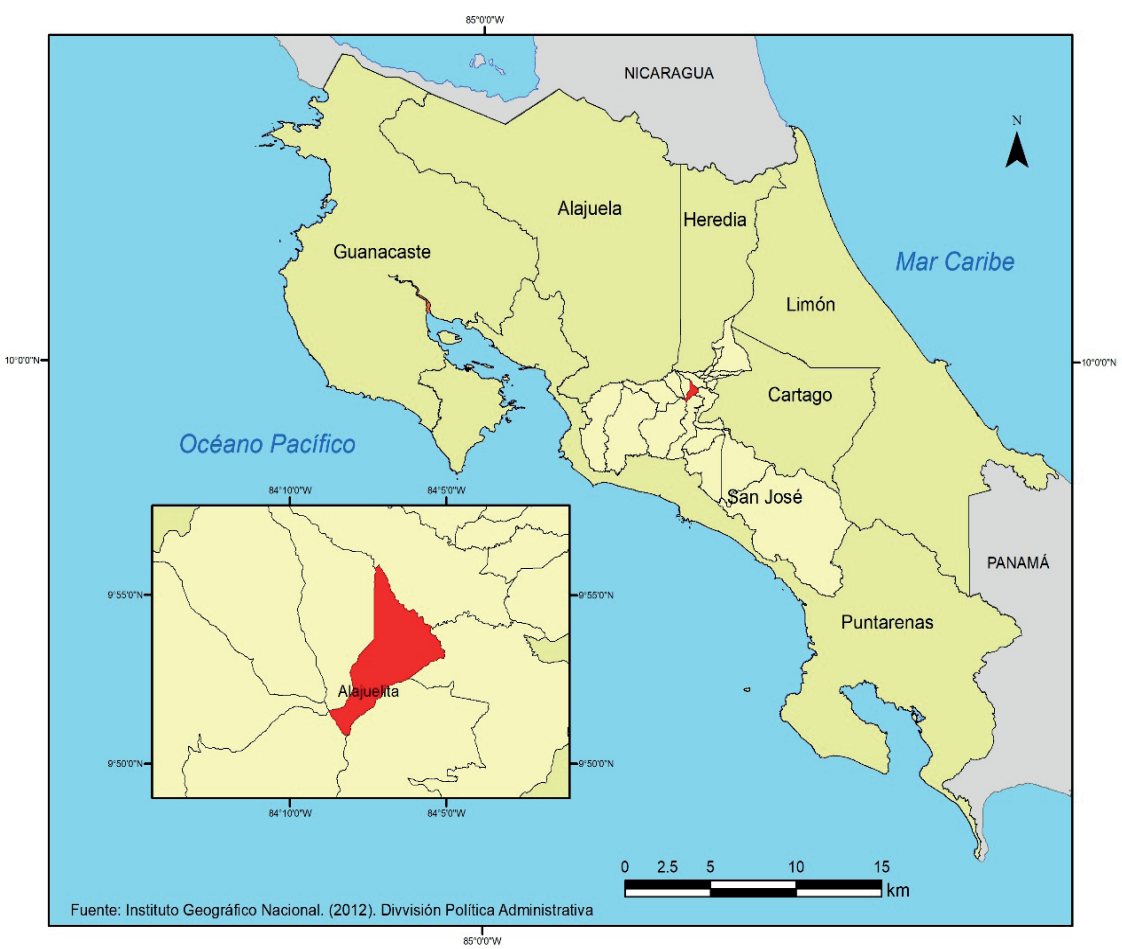

La ocupación indiscriminada de tierras por pobladores e infraestructura diversa, el deterioro ambiental que ello origina y controles municipales escasos, entre otros, ha dado como resultado el que en este cantón se 
registren problemas crecientes por activación de amenazas naturales tales como inundaciones y deslizamientos. Desde el 6 de setiembre de 1971 hasta el 8 de noviembre del año 2010, esta localidad acumuló 119 sucesos por inundación. (ídem). (Sistema de Información para Emergencias. (1994). Comisión Nacional de Prevención de Riesgos y Atención de Emergencias. Sistema de Información para Emergencias (SIE). (Salgado, 1994).

Gráfico No.1 Cantón Alajuelita, población total y densidad de población por $\mathrm{Km}^{2}$, años 1963,2011 y 2015

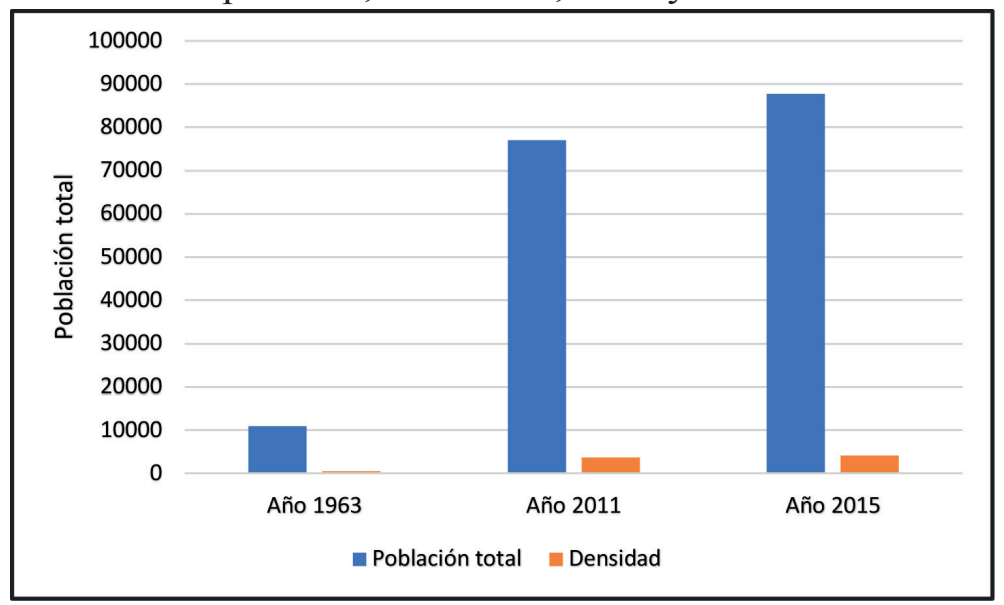

Fuente: Hernández, H. 1985. Elaboración propia con datos del INEC.go.cr

\section{Marco teórico conceptual}

La palabra BOSAI pertenece al idioma japonés, y de acuerdo con ello está formada por dos caracteres de la escritura japonesa, los cuales tienen significados diferentes, pero al unirse se complementan. El primero es BO y tiene varios significados como protección, prevención, disminución, preparación, mitigación, rehabilitación y reconstrucción. El segundo carácter es SAI el cual significa "desastre". Así, al unir estos dos caracteres, se forma una palabra que abarca todo tipo de actividades en contra de los desastres. La filosofía "BOSAI" implica acciones para disminuir los daños generados por los desastres y las medidas que se toman al respecto antes (prevención), durante (atención) y después (rehabilitación). El concepto va más allá porque es mucho más que mitigación o prevención de desastres, 
es un concepto que se asocia a la globalidad de los efectos de los fenómenos, se trata de una gestión de los riesgos. (JICA, CEPREDENAC, CNE, 2007-2012).

\section{Marco metodológico}

Una de las directrices principales descansa en el trabajo en comunidades que se encuentran bajo los efectos de amenazas naturales. Así se va trabajando con los pobladores en la construcción de herramientas prácticas y teóricas de forma que a ellos mismos se les permita conocer su ambiente natural y la dinámica de activación de las amenazas.

El BOSAI es una expresión japonesa que se ha implementado como un método en donde las comunidades, en ocasión de desastres, se organizan bajo un esquema en forma de triángulo, en donde la parte central de la figura 1 se expresa como "Reducción local del riesgo, solidaria y participativa". Se basa fundamentalmente en tres componentes ubicados en los vértices del triángulo que se expresan como ayuda a sí mismo o autoayuda, ayuda mutua o cooperación entre comunidades y ayuda institucional.

En el renglón de ayuda a sí mismo o autoayuda se organizan reuniones en comunidades con problemas por efectos de amenazas naturales, mediante la mediación de funcionarios de las municipalidades interesados en el tema y que conocen líderes comunitarios locales. A estas personas se les invita a participar como miembros activos de comités de emergencia en caseríos o vecindades que tienen problemas por amenazas naturales. (Foto 1 y 2). Al brindárseles capacitación mediante charlas técnicas y reuniones con funcionarios y personal técnico de JICA, así como con conocedores del método BOSAI, estas personas asumen papel de liderazgo en el tema ante sus vecinos por cuanto al conocer los sitios con problemas, organizan a los pobladores mediante redes de informantes, los cuales alertan y atienden de primera mano las situaciones de emergencia que pudieran presentarse. Con ello crean una primera red de atención de acuerdo con sus capacidades y posibilidades. 


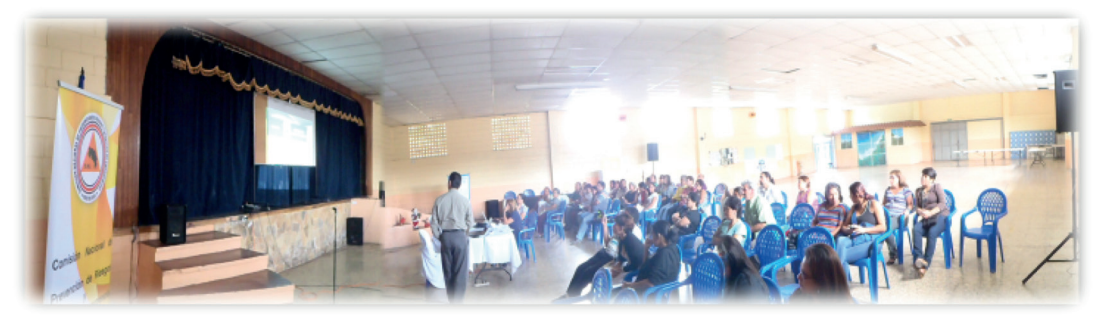

Foto 1. Reunión de pobladores, charla de capacitación. Junio 2017.

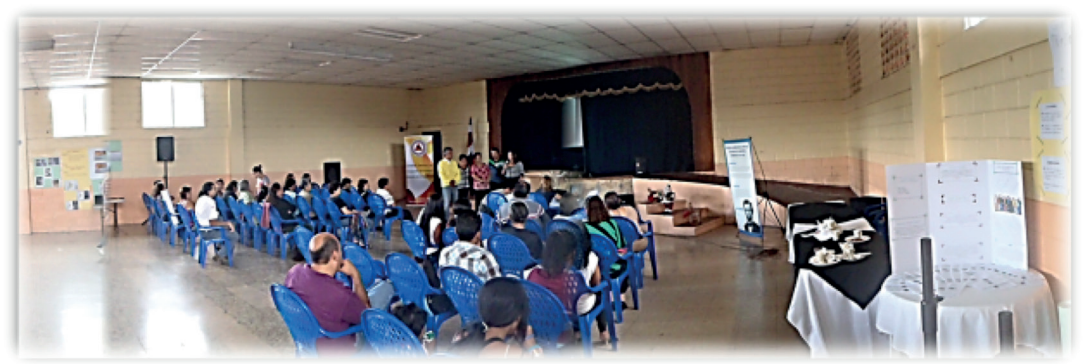

Foto 2. Reunión de líderes, charla de capacitación. Junio 2017.

De acuerdo con el postulado de autoayuda, se incentiva en los ciudadanos una autovaloración acerca de sus capacidades individuales, y de que apropiadamente asesorados, los seres humanos son capaces de afrontar de manera pragmática y exitosa las situaciones de riesgo a las que estén expuestos. La convicción paulatina que gradualmente se experimenta sobre los logros pequeños que trae la participación incipiente, así como las ventajas que se adquieren al iniciar la reducción de vulnerabilidades comunitarias a partir de concientización a lo interno de los núcleos familiares, fomenta un proceso autogestionario, en donde recurriendo a recursos propios las personas desarrollan capacidades y posibilidades.

El componente "Ayuda mutua", busca construir y enlazar los esfuerzos que, en reducción del riesgo, tienen como núcleo el entorno familiar y de autoayuda; para proyectarlo hacia la comunidad. Este procedimiento, además de extender el principio de autoayuda mediante la incorporación de otros núcleos familiares, extiende la cooperación y la solidaridad no solo en emergencias, sino también en la vida diaria, ya que fortalece relaciones vecinales. El reconocimiento y la sensibilización grupal acerca de 
cómo enfrentar situaciones de riesgo permite no solo la construcción de redes de divulgación, sino que fortalece la construcción de redes de soporte y asistencia tendientes a avanzar en la reducción de este.

El tercer componente está constituido por la "Ayuda Institucional". Esta variable reconoce la importancia de la organización comunal como fundamento básico en las tareas tendientes a reducir el riesgo. La agrupación de entidades trasciende las organizaciones públicas para extender nexos a organismos privados y a otras agrupaciones de la sociedad civil, para que en forma mancomunada, construyan y faciliten procesos de gestión y autonomía, tendientes a encaminar esfuerzos hacia una cultura que interiorice fundamentos de una cultura de la prevención dentro de un marco de participación comunitaria.

De acuerdo con los lineamientos que orientan estas capacitaciones, que se hacen también bajo la guía y conducción de la Municipalidad respectiva, se aplican los criterios de escogencia de líderes que procedan de organizaciones que contribuyen al desarrollo del cantón, tales como asociaciones de desarrollo, cooperativas, grupos organizados (Vigilancia Comunitaria) y otros que la Municipalidad proponga. Este criterio de escogencia es importante, porque así los representantes de la Municipalidad pueden dar con posterioridad seguimiento a los grupos que fueron asesorados en estas fases previas.

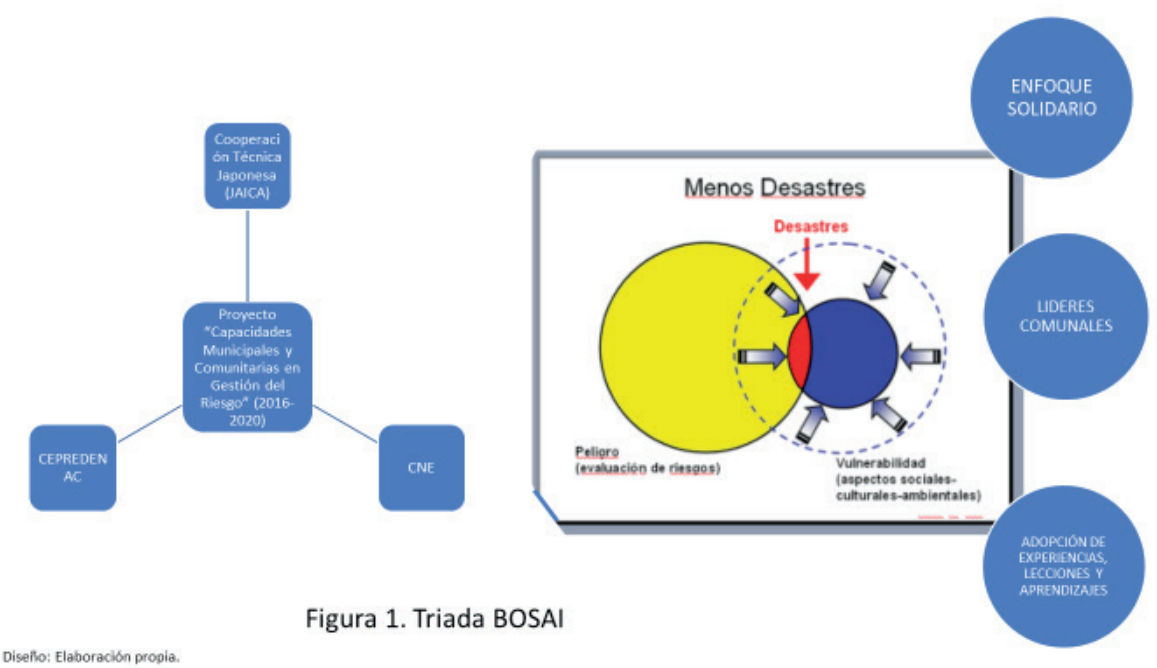




\section{Discusión de Resultados}

Al no contar este cantón con un plan regulador, las situaciones de vulnerabilidad se exacerban, ya que la aplicación de algunas de las normativas que este instrumento propondría se enfilaría hacia la delimitación de áreas de riesgo. En ausencia de tales limitaciones, es evidente qué aunque no serían una solución definitiva, al menos tanto los pobladores como la Municipalidad tendrían apoyo legal y técnico para implementar medidas de ordenamiento del territorio. De ahí que la organización comunal viene a aliviar -cuando no existe plan regulador- todas aquellas situaciones en donde su vigencia mitigaría los efectos dañinos por amenazas naturales. En el caso del cantón de Alajuelita, como resultado de estas capacitaciones existen hasta la fecha de presentación de esta ponencia; Comités Vecinales para la Atención de Emergencias, bajo las denominaciones Río Cañas, La Cascabela, Calle El Alto y Lámparas entre otros. La conformación de estos grupos ha permitido establecer vínculos de mayor cooperación y asesoría entre los ciudadanos de zonas vulnerables por amenazas naturales y los funcionarios de la Municipalidad. Esto plantea como ventaja el qué en situaciones de emergencia, los gobiernos locales no están en capacidad de atender íntegramente estos eventos, por lo que la existencia de grupos organizados y capacitados constituye un recurso importante, ya que los líderes de esos comités conocen no solo las áreas de riesgo, sino que se localizan en las cercanías de esos sitios. Tienen además el entrenamiento apropiado para el abordaje de esas situaciones, sin caer en la improvisación ni en el desorden que implicaría la no familiaridad con estos eventos. Tales procedimientos empoderan a las poblaciones por cuanto no solo se fomentan procesos sobre gestión del riesgo en el renglón de la prevención, sino que las ventajas del tratamiento apropiado sobre cómo enfrentar estas emergencias acrecienta su compromiso con los comités municipales de emergencias, a la vez que fomenta pasos tendientes a la reducción de vulnerabilidades.

\section{Conclusiones}

Mediante esta experiencia en comunidad se subsana en principio, la ausente o escasa accesibilidad que tienen algunos vecindarios, con respecto a la capacitación especializada en las etapas acerca de la gestión del riesgo por amenazas naturales. Este aspecto es de importancia trascendental por cuanto, los expertos no solo proveen información, sino que esta se 
acompaña de ejercicios y prácticas de campo en los lugares afectados. Las charlas y capacitaciones a su vez son actividades presenciales en donde la participación de los líderes les confiere reconocimientos y responsabilidades dentro del programa y de su papel en la comunidad. Es evidente que a través de las técnicas del BOSAI se ha reducido la brecha de transferencia de saberes entre entidades gubernamentales, técnicos, gobiernos locales y vecinos. Esto ha significado entre otras ventajas, el incentivar en la población una cultura de prevención y de seguimiento que tiene como evidencia el interés manifiesto por la continuidad de estas capacitaciones. El estimular la implementación de experiencias semejantes en otros ámbitos comunitarios del país permitiría sentar las bases de redes de atención permanentes y no sujetas a la temporalidad que acompaña la incidencia climática o estacional de factores de disparo de algunas amenazas naturales.

\section{Referencias}

Agencia de Cooperación Internacional del Japón (JICA), Centro de Coordinación para la Prevención de los Desastres Naturales en América Central (CEPREDENAC), Comisión Nacional de Prevención de Riesgos y Atención de Emergencias (CNE). (2007-2012). Manual par a líderes comunitarios y facilitadores en prevención de desastres bajo el enfoque BOSAI. Proyecto BOSAI-Costa Rica Fortalecimiento de Capacidades Municipal Comunitarias en Gestión del Riesgo. Preparado por D.s Salgado, G. Matamoros, S. Sánchez. Recuperado de https://www.jica.go.jp/project/all_c_america/001/materials/pdf/ manual_03.pdf)

Arroyo, L. N. (2015). Riesgos a desastres en áreas periurbanas en la Gran Área Metropolitana, consideraciones pasadas y recientes: orientaciones y alcances reales de la gestión del riesgo y el ordenamiento territorial: cantones de Aserrí, Alajuelita, Desamparados, Escazú, Santa Ana y Mora. (Informe Final Proyecto de Investigación, Escuela de Ciencias Geográficas, Universidad Nacional). Heredia, Costa Rica.

Hernández, H. (1985). Evolución territorial y principales censos de población 1502 - 1984. 1 ed. San José, CR: EUNED. Recuperado de: http://ccp.ucr.ac.cr/bvp/mapoteca/CostaRica/generales/atlas_censal/ Instituto Geográfico Nacional (2012). División Política Administrativa de Costa Rica. 
Instituto Nacional de Estadística y Censos (INEC) Población total 1963, 2011, 2015. Recuperado de http://www.inec.go.cr/content/densidadde-poblacion-alajuelita-total. Consultado 24/06/2018.

Salgado, D. (1994) Sistema de información para emergencias (S.I.E.): Aspectos generales. Comisión Nacional de Prevención de Riesgos y Atención de Emergencias. Sistema de Información para Emergencias (SIE). Recuperado de http://www.cridlac.org/digitalizacion/pdf/ spa/doc7228/doc7228.htm 